\title{
Us and them
}

\section{Our recent web focus on virology and several articles in this issue prompt us to ask how a molecular understanding of the host-pathogen interaction can lead to the development of novel strategies to combat infectious diseases.}

$\mathbf{T}$ he impact of infectious diseases on human health is obvious to anyone who has ever come down with a cold, but the consequences of infection can be far more serious and even deadly. Microbial pathogens (including bacteria, viruses, fungi and parasites) have had an important role throughout human evolution, exerting selective pressure and shaping the genetic constitution of entire populations.

The development of antimicrobials and vaccines has drastically reduced the rates of disability and death due to infectious diseases. In fact, diseases that were once widely feared, such as measles, diphtheria or tetanus, are rarely heard of in developed countries. The eradication of smallpox in the 1970s was a triumph of modern science and public health policies. An ongoing effort to eradicate another devastating disease, poliomyelitis, has already reduced infections by $99 \%$, saving an estimated five million people from permanent paralysis.

Despite such progress, infectious diseases remain a leading cause of death worldwide according to the World Health Organization (http://www.who.int/mediacentre/factsheets/fs310/en/). Though the major single cause of death in 2004 worldwide was coronary heart disease (12.2\%), this is surpassed by the combined death rates caused by lower respiratory infections, diarrhaeal diseases, HIV/AIDS and tuberculosis (16.8\%). In low-income countries, the proportion of deaths caused by infectious diseases is even higher (34\%), with neonatal infections and malaria appearing among the top ten causes of death. Even in developed countries, hospital-acquired infections are a major cause of death and increased morbidity among hospitalized patients. Although a large proportion of these infections could be avoided by changes in medical practices, their rates are going up (http://www.ahrq.gov/news/press/pr2010/qrdr09pr.htm). The rise in antimicrobial resistance, exemplified by the sharp increase in the prevalence of methicillin-resistant Staphylococcus aureus (MRSA), is a cause for alarm among public health officials.

Developing new strategies in the fight against infectious diseases thus remains an important endeavor. In The Art of War, Sun Tzu stresses that it is essential to know your enemies and to know yourself. Several reports in this issue contribute to this knowledge at the molecular or even atomic level.

On the bacterial front, Strynadka and colleagues describe the crystal structure of TagF from Staphylococcus epidermis on page 582. $\mathrm{TagF}$ is a polymerase involved in the biosynthesis of teichoic acid, a component of Gram-positive bacterial cell walls. On page 576, Hermoso and colleagues report the crystal structure of the Streptococcus pneumoniae autolysin LytC. Both papers present a wealth of structural data that provide insight into cell-wall metabolism, a process that is essential for bacterial integrity and virulence and is a potential target for antibiotic development.

Turning to parasites, finding effective antiparasite drugs continues to be an elusive task. Two groups report the structures of calcium-dependent protein kinases (CDPKs) from apicomplexan parasites (a group that includes malaria-causing Plasmodium spp). CDPKs are not present in animals and thus constitute a potential target for drugs. On page 596, Hui and colleagues present crystal structures of CDPKs in their apo and calcium-bound forms, revealing the structural reorganization involved in activation of these kinases. Merritt, Van Voorhis and colleagues report on page 602 that Toxoplasma gondii CDPK1 has a very small residue close to the ATPbinding pocket that renders the enzyme sensitive to a class of ATP analogs, known as 'bumped kinase inhibitors' because they contain a large hydrophobic moiety (a 'bump'). Moreover, the authors show that these drugs can inhibit the parasite's growth and host-cell invasion. The significance of these findings for drug development is discussed in a News and Views piece by Doerig and Billker on page 541.

Finally, viruses are the subject of a recent NSMB web focus (http:// www.nature.com/nsmb/focus/virus/index.html), featuring a Review by Das and colleagues on influenza A that appears on page 530. Influenza A viruses are responsible for seasonal flu, and newly arising variants can cause pandemics such as the recent swine flu. The authors review recent structural findings on influenza A proteins, some of which are targets for anti-flu drugs; the basis for drug resistance and potential strategies for therapeutic development are also discussed.

The web focus on virology also highlights several recent research articles in NSMB, revealing how viruses bind to and enter their target cells, the defense mechanisms mounted by the host and how viruses evade or even subvert these responses. On page 547, Andino and colleagues uncover details of the complex relationship between viruses and hosts by showing that the cricket paralysis virus targets and inhibits Argonaute 2 function in insect cells to disable antiviral responses. On page 608, Bjorkman and colleagues report the first structure of envelope gp120 from clade C HIV-1, which causes the most infections worldwide. The authors captured gp120 in complex with its receptor $\mathrm{CD} 4$ and a $\mathrm{CD} 4$-induced antibody fragment (that is, one that only recognizes gp120 when bound to CD4); unexpectedly, the structure show that the antibody makes contacts both with gp 120 and with CD4, indicating that antibody responses to HIV-1 can come at the price of autoimmunity. The implications of these findings for vaccine development are explored in a News and Views by Haynes and colleagues on page 543, which illustrates the importance of knowing yourself in battling the beast within. 